ISSN 1112-9867

http://www.jfas.info

\title{
RELATIONSHIP AND ASSOCIATION OF INFORMATION VISUALIZATION, INSIGHT AND SENSE MAKING
}

\author{
S. S. Salleh ${ }^{1, *}$, N. A. Saru ${ }^{2}$ and A. Engkamat ${ }^{1}$ \\ ${ }^{1}$ Faculty of Computer and Mathematical Sciences, UniversitiTeknologi MARA, \\ Kota Samarahan, Kuching, Sarawak, Malaysia \\ ${ }^{2}$ Faculty of Computer and Mathematical Sciences, UniversitiTeknologi MARA, \\ 40450 Shah Alam, Selangor, Malaysia
}

Published online: 17 October 2017

\begin{abstract}
In the area of Information visualization (InfoVis), the terminologies of insight and sense making have been used interchangeably. A clear difference or similarity of these terms has not been addressed and outlined. From the literature, it is anticipated that these terms involve different types of process, but the relationship with each other is still vague. Thus, in this study, the definition of InfoVis, insight and sense making were analyzed. We compiled and drew a graphical diagram of the flow of each process. Based on that we construct a generic model that consolidates and link all of identified components. As a result, we strongly proposed that sense making is an active process which is shown as a pipeline between major components within the insight process. The outcome of this study assists the researcher who is new to the field of InfoVis to become aware of the meaning of these terms.
\end{abstract}

Keywords: information visualization; insight; sense making; cognitive; data abstraction.

Author Correspondence, e-mail: ssalwa@sarawak.uitm.edu.my

doi: http://dx.doi.org/10.4314/jfas.v9i5s.33 


\section{INTRODUCTION}

Information visualization (InfoVis) is one of the impacts of the evolution of the technology advancement in representing information. By the scenario today in which the huge size of information has been gathered, the need of InfoVis becomes necessary in order to display and deal with the big data. Thus, presenting information or knowledge in the most accurate form to the targeted users is vital. InfoVishas also become a major tool for gaining insight [1-2]. InfoVisenlarges human cognition by presenting the information graphically [2-3]. This method usually engages with complicated data alteration, interpretation, and communication techniques. It can be very complex [4] as it connects human's visual insight to recognize trends, patterns and uncommon occurrences in datasets [5]. Upon that, to present information accurately requires an understanding of how it is being perceived cognitively.

In the area of visualization, InfoVis, insight and sense making occur in most literature interchangeably. Most of the time these three terms have been defined based on individual context. A clear difference or similarity of these terms has not been addressed and outlined. Based on this regard, this study aims to assist researcher who is new to the field of InfoVis to become aware of the three important terminologies that always found in the most of their foundational literature.

In this study we review definitions given by researchers and then we draw the flow of processes of these three terminologies from selected articles. Based on that, we construct the acquaintance of the basic key elements of the processes and form a relationship of identified components.

This paper is divided into seven sections. It starts with the first section which covers on the introduction. The second section comprises of the study motivation, problem statement, objectives and the significance of this study. The third section presents briefly on the literature of the three terms. Section four is focusing on the methodology and the details of the InfoVis, insight and sense making process. Finally, in section five, generic model is constructed and the discussion of the model is also presented. And the last section which is section six is the conclusion of the overall study. 


\section{STUDY MOTIVATION AND OBJECTIVE}

Knowledge is a theoretical or practical understanding of a subject which consists of a relationship between information and facts [6]. It is commonly represented by symbols. Its purpose is to facilitate inference from the knowledge elements before creating new ones. The representation of knowledge must be in the form that understood by humans [7]. Visualizing information using symbols, shapes and other graphic representations must comply with the purpose of knowledge formation. As that relates to the interpretation of knowledge which goes through certain cognitive processes.

InfoVis, insight and sense making have been discussed in several studies. Literature characterized these terms independently based on their context of study. In our hypothesis, we anticipate that there must be a certain linkage among them. Apart from that, we wanted to see its association in a bigger perspective. Thus, we attempt to address this association and relationship by reviewing the literature pertaining to these three cognitive flow of processes.

The main objectives of this study are as the followings. The first objective is to review, select and extract basic flow of a defined concept of insight, sense making and InfoVis. The second objective is to analyze the relationship between said concepts. And the third objective is to consolidate and synthesize a relationship model of the three elements in generic form.

\section{BACKGROUND}

InfoVis is closely related to perception. Generally, the perception is defined as a process in which human's stimulation is converted into systematic knowledge. Human manage and understand the information using sensory receptors and this is called as perception process. Visual perception facilitates sense making. In general, sense making refers to how human find structure in an ambiguous situation, involving informational, communicational and computational processes [8]. A sense making is simply defined as "making sense of things". In [9] defined it more comprehensively as "a motivated and continuous effort to understand the connections".

Visual perception also comes from visual information that provide an insight of certain matters. The process of insight includes how the users see the data that is visually represented through their sight or vision.Insight can be generally described as a human cognition to 
achieve the meaning of the data that is viewed in a logical form. In previous research[10]mentioned that the purpose of InfoVis is to provide effective tools and methods in gaining insight as well as to give better knowledge of a dataset [10]. $\operatorname{In}[6]$ stated that insights can be recorded in a form of notes or list. The changes in the notes refer to the insight actions. He added that users clearly indicate visualizations that are derived from their insights into visual insight actions. Insight can also be facilitated through making connections between digital representations like a graph or numerical dataset, or into scientific phenomena they are intended to represent [11-12].

According to [5], insight and sense making are interrelated in which to understand the procedural aspects of insights, they found that understanding sense making is also important. Interesting discussion on insight and sense making has been found out in several studies, but the similarity and differences of the procedural aspects have not been discussed. Therefore, in order to look into this matter, we conducted a review on it.

\section{METHODOLOGY}

Collectively 27 published papers consist of 10 journal articles, 9 conference papers and 8 technical reports and 3 book chapters have been reviewed in this study. The databases that are mostly used are from IEEE, ACM, Springer and SAGE. From all of these documents, some analyses are made to identify the concept of insight, InfoVis, sense making process as well as on how they relate and associate with each other. The focus has been given to articles that presented a definition or discussion on the process and flow significantly. Selected definition from few articles are presented in the following section.

The definitions were compiled and plot in the form of graphical flow. The purpose is to easily discover its similarities and differences. Through the manual observation, we examine each of the general basic flow among the identified components. Based on the graphical diagram, we will be able to locate common flow and sequences if those were exist. In later sections, we present a discussion of prevalent attributes discover from the analysis.

In this section, analysis of five definitions of InfoVis, eight flow of processes of insight, and five flow of processes of sense making are presented. Table 1 presents the summary of the statement of each definition. 
Table 1. The compilation InfoVis definition

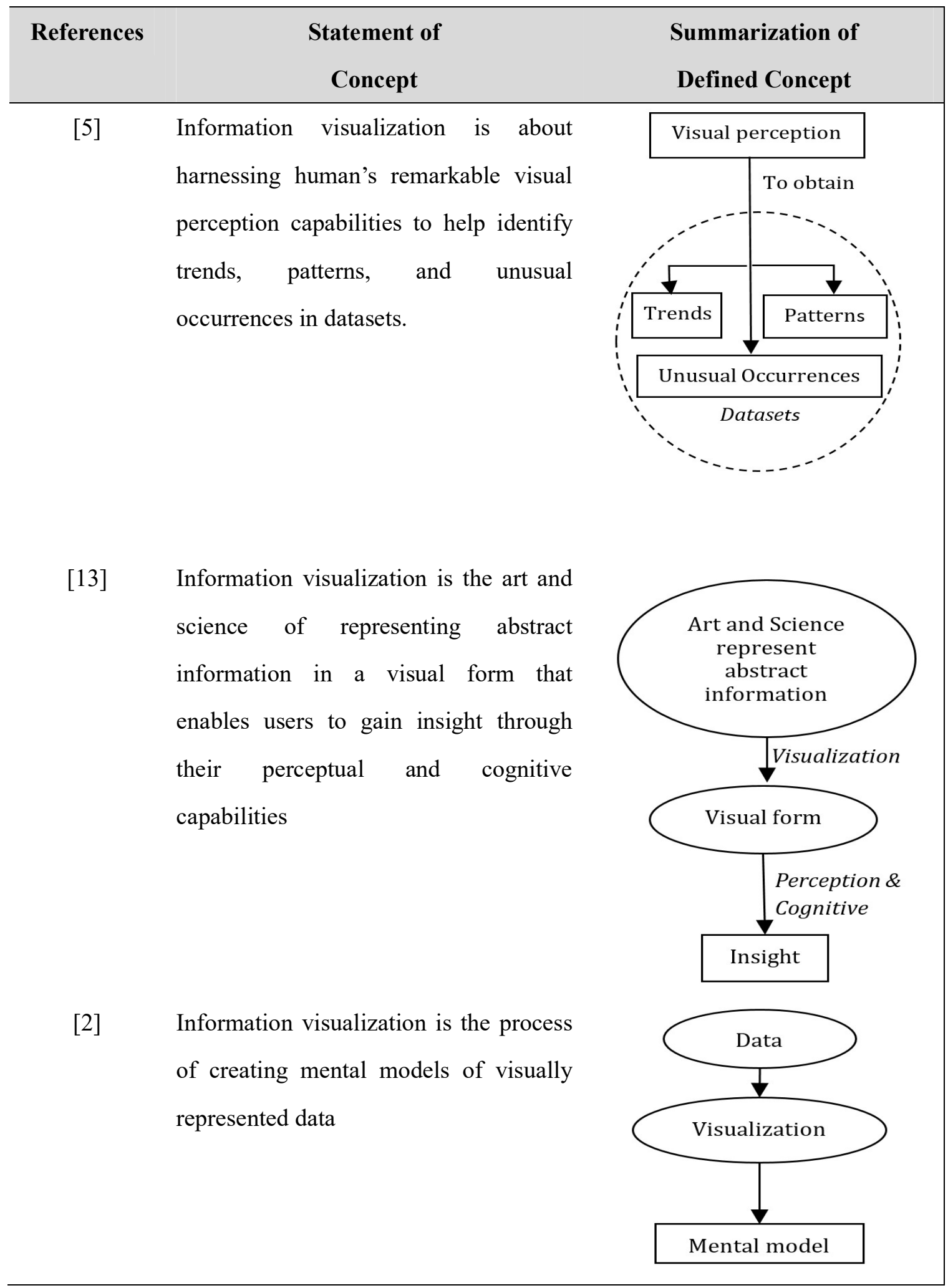


[14] Information visualization is a way to present data graphically to amplify human cognition.

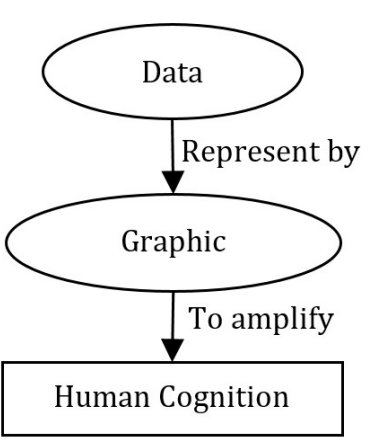

Information visualization is a tool to

visually represent abstract concepts that are not inherently spatial, since the associated data have no clear physical representation.

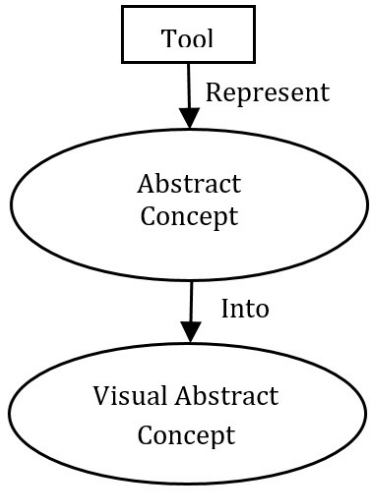

From the table, in[5] stated that InfoVis is used to gain occurrence in a dataset which we classify as insight. In $[2-3,13]$ used different terms in their definition as they stated their final outcome of InfoVis is a mental model, human cognition and visual abstract concept respectively. Definition discussed by [11-12] as given in the prior section, classify these terms as insight. Therefore, in [3, 5, 14-15] are actually similar. In [1] discussed the theory in the context of philosophical approach, but when we summarized it in a form of graphical representation it is still similar to the rest.

In the next table which is Table 2, the summary of defined flow of process of insight and its remark is shown. Similar to Table 1, all of the definition that are presented in the table is arranged based on the year which the study has been conducted

Table 2.The compilation of insight process flow

\begin{tabular}{clc}
\hline References & $\begin{array}{c}\text { Concept of Insight That is Constructed } \\
\text { in Authors' Definition }\end{array}$ & Summary of Defined Concept \\
\hline$[16]$ & An individual observation about the data & Observe \\
& by the participant. & $\checkmark$ \\
& & Data \\
\hline
\end{tabular}


Meaning

[17] Insight is complex, deep, qualitative, unexpected and relevant.

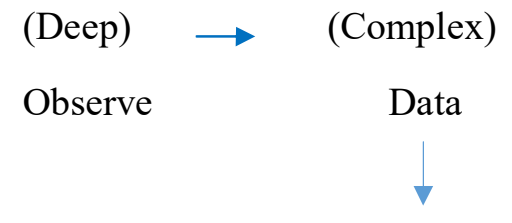

Meaning

(Qualitative, unexpected and relevant)

[5] The Insight is not only an end result or simple discovery of hidden truth, but also an intermediate state in the iterative and cyclic procedure of sense making and invention

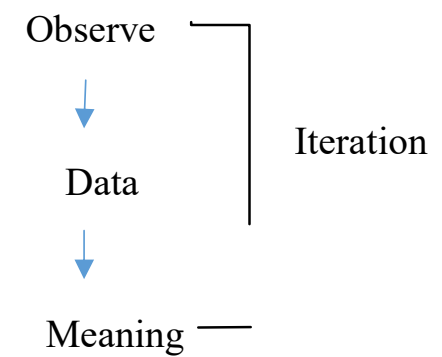

[2] The Insight comes from human powers of Observe deduction and perception and from mapping observations against mental Data models of what is being seen

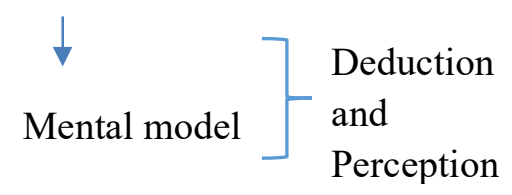

[18] Insight is a form of learning that builds a relationally semantic knowledge base

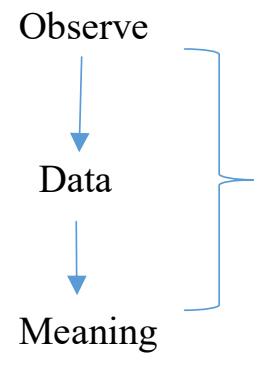

Problem-solving through a variety problem-solving and reasoning heuristics. and reasoning heuristic techniques 


[19] Insight is related to the concept of
exploration. It is usually assumed that
exploration is necessary for gaining
insight.
[9]
Insight is a discontinuous discovery, a
non-obvious revision to a person's mental
model of a dynamic system, resulting in a
new set of beliefs that are more accurate,
comprehensive, and useful.
[3]
Insight is the ultimate goal of any
visualization tool; to gain insight, user Involve in activities
engages in activities that help them make
sense of the domain as represented by the Sense making
(Visualization tools)

In [16] defined insight as a sequential process.Similarly,in [17] also stated that it comprises of three main components, but in addition to that it focused on the characteristics of insight that detailing on the observation, data and meaning. In [5] defined insight similar like the previous two researchers, but he added that it is actually is a cyclic process. In [2] had a similar flow of the process, but he emphasized on the last component which is on the mapping observation into meaning that involve a mental model approach. Study by [18] emphasized that insight is a learning process and the process is growing. It discussed much on problem-solving and reasoning heuristic techniques involve along the process of insight. In [17] defined similar idea to [16]. Study by [18] is contradict from another school of thoughts where they defined insight as a disjoint and dynamic process. Its definition is obviously similar to [18]. Study by [3] support the study of $[18,21]$. It imposed an idea that there must be a group of activities involves, where sense making were developed from these activities. Deduction of the sense 
making compose an insight. From this, it found that basically (i) insight is the finite goal; (ii) the process to gain insight is growing and in sequence; (iii) insight comprises of three major components. Interestingly, to gain insight it must go through (i) some observations; or (ii) activities; or (iii) usage of tools and technique. It wrap these and named it as visual scheme. Next, Table 3 compiles the graphical flow of the sense making process.

Table 3. Summary sense making process

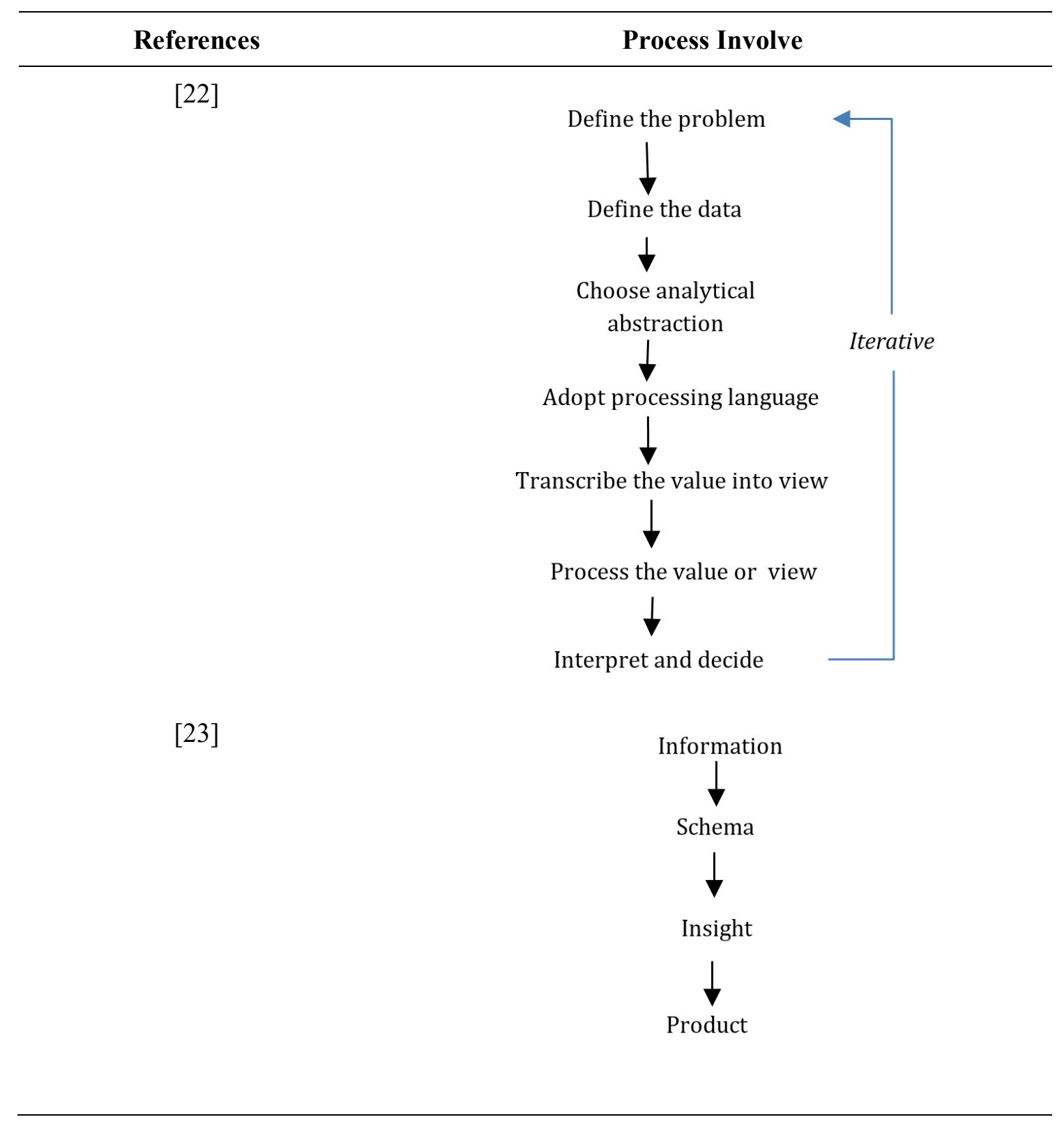


[20]

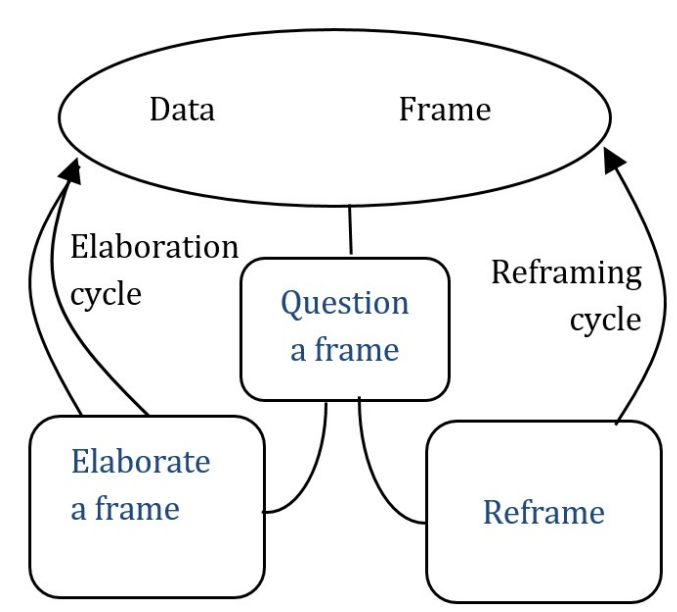

Preserve

[13]

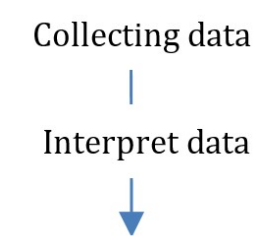

Plan the next step

[20]

Building
$\mid$
Elaborating
$\mid$
Preserving
$\mid$
Questioning
$\mid$
Revising
$\downarrow$
Replacing frame

Studies by [22] presented the flow of sense making in the perspective of theoretical process, while in[23] discussed it in the technical approach. In [13] also presented it in a plain and simple flow. On the other hand, although there are only two steps is discussed by [9], it however has been elaborated in detail. The sense making process defined by [20] is quite similar to [9], but steps in their latest model have been enhanced as a well been arranged in sequence of flow. We point out our stand that sense making is an iterative process. Even 
though it has not been mentioned clearly in some of the reviewed articles, but it somehow we realized that sense making is actually a collection of actions. It is therefore concluded as an active process. The process is accumulated as it collects meanings. The collection of meanings was produced by iterations of the process and at the end it created an insight.

\section{RESULTS AND DISCUSSION}

Based on the findings stated in the previous section, we formulate and construct a model. The model comprises of the flow of processes that associates InfoVis, insight and sense making as a whole. It generalizes human cognition process involve in gaining insight from InfoVis where sense making is the major cycle within it. The model is depicted in Fig. 1. The input in this model is data and the output is the meaning deriving from a mental model. Visual schema is used to represent data in the form of table, charts, shapes, arrangement of flow, text and graphical diagram. The schema is re-documentation or prearranged collection of the information that is used to represent the conclusion [4]. Schema presents the data in a simplified form so that it makes the task of transcription and interpretation become easier.

According to [7], in order to have a better insight of information visualization, we need to go through the process of sense making first. Therefore, we associate sense making as the cyclic pipeline within the insight. The pipelines in the model shows that the sense making pipelines are an active flow. We agree with $[9,24]$ who stated that sense making can be defined as creativity, curiosity, comprehension, mental modeling procedures and situation awareness. The followings are further descriptions of each pipeline:Transcribe-It is performed after problem and data are defined, an analysis and visualization abstraction is chosen. In this process a new visualization state is generated after the visualization pipeline has been executed [21]. User can see the visualization at this stage since it copies out the data into view.ii. Interpret-It takes place after several steps has been performed and several improvements like zooming, rotation and filtration of the data abstraction done. The outcome will affect the interest of the user. The better the outcome, the more users' attention would be and precise translation will take place. 


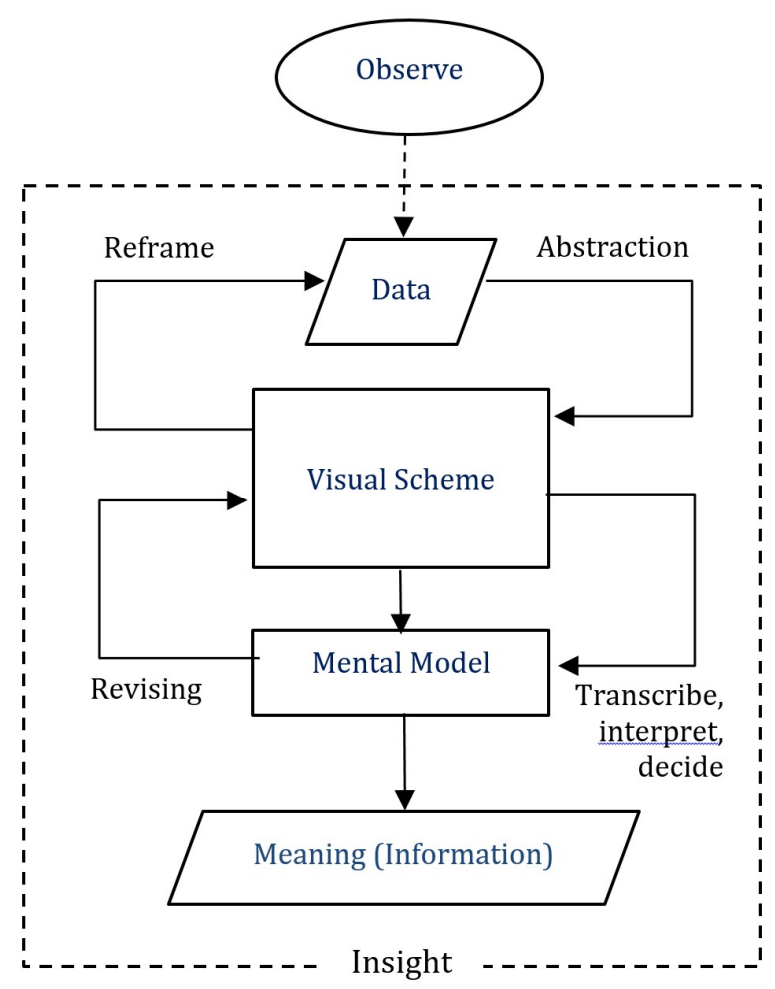

Fig.1. Element in InfoVis

iii. Decide-After the users' attention is obtained, the task of producing insight will be taken place. The user will decide either to take their interpretation of visualization as a meaning of the data or not.In [25] classified insight process in two major classes, which are (i) basic and (ii) comparative insight. Each of the class comprises of four types of activities. Basic visual insights comprise of Read Value (Va), Identify Extrema (Ex), Characterize Distribution (Di) and Describe Correlation (Co) process. While comparative insights are higher-order insights that compare the results of the four types of basic insights. Accordingly, in [25] stated that there are four types of them: Compare Values (VC), Compare Extrema (EC), Compare Distribution (DC) and Compare Correlation (CC). There was a detail experiment and discussion on insight made by [25].However, in the context of this study, we define the process up to this stage only.

Meaning is known as the goal or output of InfoVis. Meaning is the understanding or user's perception through the data that is visually represented. In the context of this study, it is also referred as information. The model that we constructed, consistent with idea imposed by [4, 23 ] as they also stressed out that the product of information visualization is obtained through 
sense making process after the insight is gained [13].Herewith, InfoVis is actually is a noun that identifies a group process.

\section{CONCLUSION}

In this study, the definition of InfoVis, insight and sense making were reviewed. We compiled and drew a graphical diagram of the flow of each process. From the findings, we conclude that insight is the process and sense making is the pipeline within InfoVis. In the other hand, it can be said that the InfoVis is a tool for gaining insight. The proposed model has given a comprehensive understanding on what are insight and sense making, how it associates in InfoVis as well as the flow of respective activities.

\section{ACKNOWLEDGEMENTS}

This study is funded by UiTM IRAGS Grant (600/IRAGS 5/3 (5/2015).

\section{REFERENCES}

[1] Kalawsky R. S. Gaining greater insight through interactive visualization: A human factors perspective. In E. A. Seinstra (Ed.), Trends in interactive visualization.London: Springer, 2009, pp. 119-154

[2] Eppler M. What is an effective knowledge visualization?Insights from a review of seminal concepts. In F. Marchese, \& E. Banissi (Eds.),Knowledge visualization currents.London: Springer Verlag, 2013,pp. 3-12

[3] Faisal S, Blandford A, Potts H W. Making sense of personal health information: Challenges for information visualization. Health Informatics Journal, 2013, 19(3):198-217

[4] Plaisant C, Fekete J D, Grinstein G. Promoting insight-based evaluation of visualizations: From contest to benchmark repository. IEEE Transactions on Visualization and Computer Graphics, 2008, 14(1):120-134

[5] Yi J S, Kang Y A, Stasko J T, Jacko J A. Understanding and characterizing insights: How do people gain insights using information visualization? In Workshop on Beyond Time and Errors: Novel Evaluation Methods for Information Visualization, 2008, pp. 1-6

[6] Gotz D, Zhou M X. Characterizing users' visual analytic activity for insight provenance.In 
IEEE Symposium on Visual Analytics Science and Technology, 2008, pp. 123-129

[7] GouL, Zhang X, Luo A, Anderson P F.SocialNetSense: Supporting sensemaking of social structural features in networks with interactive visualization. In IEEE Symposium on Visual Analytics Science and Technology, 2012, pp. 133-142

[8] Andrews K, Lessacher M. Liquid diagrams: Information visualisation gadgets. In 14th International Conference Information Visualisation, 2010, pp. 104-109

[9] Klein G, Moon B, Hoffman R R. Making sense of sensemaking 1: Alternative perspectives. IEEE Intelligent Systems, 2006, 21(4):70-73

[10] Zuk T, Schlesier L, Neumann P, Hancock M S, Carpendale S. Heuristics for information visualization evaluation. In ACM AVI Workshop on Beyond Time and Errors: Novel Evaluation Methods for Information Visualization, 2006, pp. 1-6

[11] Elmqvist N, Moere AV, Jetter HC, Cernea D, Reiterer H, Jankun K. TJ. Fluid interaction for information visualization. Information Visualization, 2011, 10(4):327-340

[12] Eppler M J. What is an effective knowledge information visualization. In 15th International Conference of Information Visualization, 2011, pp. 349-354

[13] Robertson G, Czerwinski M, Fisher D, Lee B. Selected human factors issues in information visualization. Reviews of Human Factors and Ergonomics, 2009,5(1):41-81

[14] Hamzah M, Sobey A, Koronios A.Supporting decision making process with information visualisation: A theoretical framework. In 2nd IEEE International Conference onInformation Management and Engineering, 2010, pp. 267-271

[15] Robinson N, Shapcott M.Data mining information visualisation-Beyond charts and graphs. In 6th IEEEInternational Conference on Information Visualisation, 2002, pp. 577-583

[16] Saraiya P, North C, Duca K. An insight-based methodology for evaluating bioinformatics visualizations. IEEE Transaction on Visualization and Computer Graphics,2005, 11(4):443-456

[17] North C. Toward measuring visualization insight. IEEE Computer Graphics and Applications, 2006, 26(3):6-9

[18] Chang R, Ziemkiewicz C, Green T M, Ribarsky W.Defining insight for visual analytics. IEEE Computer Graphics and Applications, 2009, 29(2):14-17

[19] Pohl M, Wiltner S, Miksch S.Exploring information visualization-Describingdifferent 
interaction patterns.In 3rd ACM BELIV'10 Workshop: Beyond Time and Errors: Novel Evaluation Methods for Information Visualization, 2010, pp. 16-23

[20] Klein G, Jarosz A.A naturalistic study of insight. Journal of Cognitive Engineering and Decision Making, 2011, 5(4):335-351

[21] Klein G, Moon B, Hoffman R R.Making sense of sensemaking 2: A macrocognitive model. IEEE Intelligent Systems, 2006, 21(5):88-92

[22] Chi E H, Card S K. Sensemaking of evolving web sites using visualization spreadsheets.In IEEE Symposium on Information Visualization, 2000, pp. 18-25

[23] Pirolli P, Card S. The sensemaking process and leverage points for analyst technology as identified through cognitive task analysis. In International Conference on IntelligenceAnalysis, 2005, pp. $2-4$

[24] Mikkelsen C, Johansson J, Rissanen M. Interactive information visualization for sensemaking in power grid supervisory control system. In 15th IEEEConference on Information Visualisation, 2011, pp. 119-126

[25] Yang H, Li Y, Zhou M X. Understand users' comprehension and preferences for composing information visualizations. ACM Transactions on Computer-Human Interaction, 2014, 21(1):1-30

\section{How to cite this article:}

S. S. Salleh, N. Ahmad Saru, A. Engkamat, Relationship And Association Of Information Visualization, Insight And Sense Making, Appl. Sci., 2017, 9(5S), 466-480. 